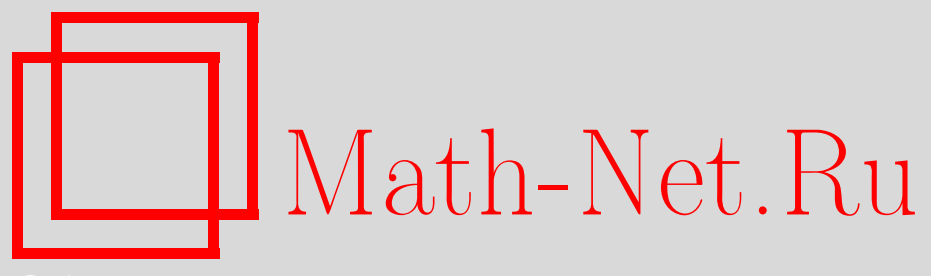

Д. Сондоу, С. А. Злобин, Интегралы по многогранникам, кратные дзета значения и полилогарифмы, и константа Эйлера, Матем. заметки, 2008, том 84, выпуск 4, 609626

DOI: https://doi.org/10.4213/mzm6140

Использование Общероссийского математического портала Math-Net.Ru подразумевает, что вы прочитали и согласны с пользовательским соглашением http://www . mathnet.ru/rus/agreement

Параметры загрузки:

IP : 54.198 .187 .58

26 апреля 2023 г., 12:53:18

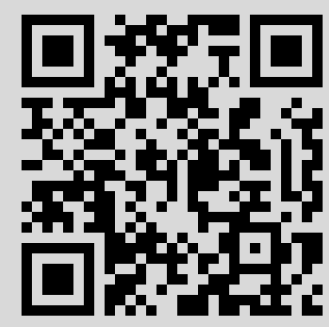


Том 84 выпуск 4 октябрь 2008

УДК 517.51

\section{Интегралы по многогранникам, кратные дзета значения и полилогарифмы, и константа Эйлера}

\section{Дж. Сондоу, С. А. Злобин}

Пусть $T$ - треугольник с вершинами $(1,0),(0,1),(1,1)$. Рассматриваются некоторые интегралы по $T$, один из которых был вычислен еще Эйлером. Приводятся выражения для этих интегралов как в виде линейной комбинации кратных дзета значений, так и в виде полинома относительно обычных дзета значений. Получены асимптотические разложения интегралов и сумм некоторых кратных дзета значений с постоянным весом. Также представлены соответствующие выражения для константы Эйлера. В заключительном пункте вычисляются более общие интегралы, один из которых - повторный интеграл Чена (Дринфилда-Концевича), по некоторым многогранникам, являющимся многомерными аналогами треугольника $T$. Это приводит к соотношению, связывающему некоторые кратные полилогарифмические значения и кратные дзета значения.

Библиография: 17 названий.

1. Введение. Пусть $T$ - треугольник, заданный выражением

$$
T:=\left\{(x, y) \in[0,1]^{2} \mid x+y \geqslant 1\right\}
$$

с вершинами $(1,0),(0,1),(1,1)$. В данной работе мы изучаем интеграл по треугольнику $T$

$$
I_{n}:=\iint_{T} \frac{(-\ln x y)^{n}}{x y} d x d y
$$

для $n=-1,0,1,2, \ldots$. Мы также рассматриваем интегралы по нескольким многогранникам, которые являются многомерными аналогами треугольника $T$.

Эйлер вычислил повторный интеграл, эквивалентный $I_{0}$, и получил, что

$$
\begin{aligned}
I_{0} & =\iint_{T} \frac{d x d y}{x y}=\int_{0}^{1} \frac{1}{x} \int_{1-x}^{1} \frac{d y}{y} d x=\int_{0}^{1} \frac{-\ln (1-x)}{x} d x \\
& =\int_{0}^{1} \sum_{r=1}^{\infty} \frac{x^{r-1}}{r} d x=\sum_{r=1}^{\infty} \frac{1}{r^{2}}=\zeta(2) .
\end{aligned}
$$

Интегрируя по частям, он вывел формулу (22), которую использовал для вычисления $\zeta(2)$ с точностью до шестого знака после запятой, см. [1; раздел 1.2], [2; с. 43-45]. 
Мы обобщаем результат Эйлера на случай $n=0,1,2, \ldots$, показав, что $I_{n}$ равен целой линейной комбинации кратных дзета значений

$$
\zeta\left(s_{1}, \ldots, s_{l}\right):=\sum_{n_{1}>n_{2}>\cdots>n_{l}>0} \frac{1}{n_{1}^{s_{1}} \cdots n_{l}^{s_{l}}}
$$

$c$ весом $s_{1}+\cdots+s_{l}=n+2$. Мы также представим $I_{n}$ в виде полинома относительно обычных дзета значений.

Теорема 1. Пусть $n \geqslant 0-$ целое число.

(i) Тогда

$$
I_{n}=n ! \sum_{k=0}^{n} \zeta\left(n-k+2,\{1\}_{k}\right),
$$

где через $\{1\}_{k}$ обозначен набор $1,1, \ldots, 1$ ( $k$ раз).

(ii) Кроме того, $I_{n}$ является полиномом в явной форме от многих переменных с рациональными коэфбициентами относительно значений дзета функции Римана $\zeta(2), \zeta(3), \ldots, \zeta(n+2)$.

Теорема 1, следствие 1 и лемма 1 были получены вторым автором в [3]. Доказательство этих результатов приведено в п. 2, вместе с явными формулами. Рассмотрены следующие примеры:

$$
\begin{aligned}
& I_{0}=\zeta(2), \\
& I_{1}=\zeta(3)+\zeta(2,1)=2 \zeta(3), \\
& I_{2}=2(\zeta(4)+\zeta(3,1)+\zeta(2,1,1))=\frac{9}{2} \zeta(4), \\
& I_{3}=6(\zeta(5)+\zeta(4,1)+\zeta(3,1,1)+\zeta(2,1,1,1))=36 \zeta(5)-12 \zeta(2) \zeta(3) .
\end{aligned}
$$

Случаи $n=0,1,2$ особенно простые.

СлЕДСТВИЕ 1. При $n=0,1,2$ интеграл $I_{n}$ является рационалъным кратным числа $\zeta(n+2)$.

При $n=0,1$ это также следует из формул, полученных Бейкерсом [4] для $\zeta(2)$ и $\zeta(3)$ в виде интегралов по единичному квадрату

$$
S:=[0,1]^{2} .
$$

Именно, замена переменных $x=X, y=1-X Y$ преобразует как $I_{0}$ в

$$
I_{0}=\iint_{T} \frac{d x d y}{x y}=\iint_{S} \frac{d X d Y}{1-X Y}=\zeta(2),
$$

так и $(1 / 2) I_{1}$ в

$$
\begin{aligned}
\frac{1}{2} I_{1} & =\frac{1}{2} \iint_{T} \frac{-\ln x y}{x y} d x d y=\iint_{T} \frac{-\ln x}{x y} d x d y \\
& =\iint_{S} \frac{-\ln X}{1-X Y} d X d Y=\frac{1}{2} \iint_{S} \frac{-\ln X Y}{1-X Y} d X d Y=\zeta(3) .
\end{aligned}
$$

Приведем схему доказательства теоремы 1 , но сначала сформулируем следующую лемму. 
ЛЕмма 1. Если $k \geqslant 0$ u $l \geqslant 0$ - целые числа, то

$$
I_{k, l}:=\iint_{T} \frac{(-\ln x)^{k}(-\ln y)^{l}}{x y} d x d y=k ! l ! \zeta\left(l+2,\{1\}_{k}\right) .
$$

Кроме того, если $l \geqslant 1$, то

$$
J_{k, l}:=\int_{0}^{1} \frac{(-\ln (1-x))^{k}}{1-x}(-\ln x)^{l} d x=k ! l ! \zeta\left(l+1,\{1\}_{k}\right) .
$$

Разлагая $(-\ln x-\ln y)^{n}$ в ряд, мы сразу получим утверждение (i). Чтобы закончить доказательство леммы, покажем, что $(l+1) I_{k, l}=J_{k, l+1}$, и затем вычислим интеграл $J_{k, l}$. Утверждение (ii) теоремы 1 доказывается с помощью формулы, полученной в [5], где $J_{k, l}$ выражено через обычные дзета значения.

В качестве примера получим явную версию результата, доказанного в [6].

СлЕДСТВИЕ 2. Если $n \geqslant 2 u k \geqslant 0$, то кратнъе дзета значения $\zeta\left(n,\{1\}_{k}\right)$ можно представить в явном виде как полином от многих переменных с рациональными коэффичиентами относительно обычных дзета значений $\zeta(2), \zeta(3), \ldots, \zeta(n+k)$.

Лемма 1 также дает простое доказательство частного случая теоремы двойственности для кратных дзета значений (см., например, [1; раздел 2.8]).

СлеДСтвиЕ 3. Если $k \geqslant 0 u l \geqslant 0$, mo

$$
\zeta\left(k+2,\{1\}_{l}\right)=\zeta\left(l+2,\{1\}_{k}\right) .
$$

Например, $\zeta(2,1)=\zeta(3)$ и $\zeta(2,1,1)=\zeta(4)$. С помощью этих равенств мы дадим второе доказательство следствия 1. Однако в отличие от случаев $n=0$ и 1 утверждение следствия 1 в случае $n=2$ мы не можем доказать без использования теоремы 1.

Используя численные результаты и примеры, такие как (3), мы сделаем следующее

ПРеДПОЛОЖЕНИЕ 1. Интеграл $I_{n}$ не является рациональным кратным числа $\zeta(n+2)$ при $n>2$.

Это утверждение не было доказано ни для одного значения $n$. Однако с помощью теоремы 1 , (ii) мы можем привести условное доказательство для всех $n=3,4, \ldots$ при стандартном предположении (см., например, [7; введение]).

Теорема 2. Если числа $\pi, \zeta(3), \zeta(5), \zeta(7), \zeta(9), \ldots$ алгебраически независимы над полем рачиональных чисел, то предположение 1 верно.

С помощью леммы, которая дает асимптотическое разложение для коэффициентов ряда Тейлора некоторых мероморфных функций (лемма 2), мы оценим $I_{n}$ при больших $n$.

ТЕОРема 3. Имеет место асимптотическая эквивалентность

$$
I_{n} \sim 2 n !, \quad n \rightarrow \infty .
$$

Более точно, имеет место следующее асимптотическое разложение:

$$
\frac{I_{n}}{n !} \approx 2+\frac{6}{2^{n+2}}+\frac{20}{3^{n+2}}+\frac{70}{4^{n+2}}+\cdots, \quad n \rightarrow \infty,
$$

где числитель в $k$-м члене имеет вид $\left(\begin{array}{c}2 k \\ k\end{array}\right)$ nри $k=1,2, \ldots$. 
Это в свою очередь дает оценку для суммы кратных дзета значений $\zeta\left(m-k,\{1\}_{k}\right)$ с постоянным весом $m$.

СлЕдствиЕ 4. Среднее кратных дзета значений $\zeta(m), \zeta(m-1,1), \ldots, \zeta\left(2,\{1\}_{m-2}\right)$ асимптотически стремится $\kappa 2 / m$ при т стремящемся $\kappa$ бесконечности. Точнее, имеет место следующее асимптотическое разложение:

$$
\sum_{k=0}^{m-2} \zeta\left(m-k,\{1\}_{k}\right) \approx 2+\frac{6}{2^{m}}+\frac{20}{3^{m}}+\frac{70}{4^{m}}+\cdots, \quad m \rightarrow \infty .
$$

Еще одно применение теоремы 3 дает следующий любопытный результат.

СлеДСТвиЕ 5. Ряд

$$
\sum_{n=0}^{\infty}(-1)^{n} \frac{I_{n}}{n !}
$$

расходится, но является суммируемым по Абелю, и его сумма равна 1/2.

Пойдем "вниз" от $I_{0} \mathrm{~K} I_{-1}$.

Вопрос. Можно ли вычислить интеграл

$$
I_{-1}=\iint_{T} \frac{d x d y}{x y(-\ln x y)}=1.7330025 \ldots,
$$

используя более известные константы?

Удивительно, но оказалось, что $I_{-1}$ содержит в себе все интегралы $I_{0}, I_{1}, I_{2}, \ldots$ (и, следовательно, все кратные дзета значения $\zeta\left(m,\{1\}_{k}\right)$ при $m \geqslant 2$ и $\left.k \geqslant 0\right)$.

Теорема 4. Если li - логарифмическая целая функиия, то

$$
I_{-1}=\sum_{n=0}^{\infty}(-1)^{n} \frac{I_{n}}{(n+1) !}+\int_{0}^{1} \frac{\operatorname{li}\left(x-x^{2}\right)}{x} d x+1 .
$$

(Ср. сходящийся ряд здесь и расходящийся ряд в следствии 5.)

Теперь мы выразим двойной интеграл $I_{-1}$ через простые интегралы, один из которых содержит обобщенный биномиалъный коэффициент

$$
\left(\begin{array}{l}
s \\
t
\end{array}\right):=\frac{\Gamma(s+1)}{\Gamma(t+1) \Gamma(s-t+1)} .
$$

ПреДЛОЖенИЕ 1. Имеют место следующие интегралъные формулы для $I_{-1}$ :

$$
I_{-1}=\int_{0}^{\infty}\left(1-\frac{1}{\left(\begin{array}{c}
2 t \\
t
\end{array}\right)}\right) \frac{d t}{t^{2}}=\int_{0}^{1} \ln \left(1+\frac{\ln (1-x)}{\ln x}\right) \frac{d x}{x} .
$$

Разлагая первый интеграл в степенной ряд, мы видим, что $n$-й коэффициент содержит интеграл $I_{n}$.

Tеорема 5. Если $0<|t|<1$, mo

$$
\left(1-\frac{1}{\left(\begin{array}{c}
2 t \\
t
\end{array}\right)}\right) \frac{1}{t^{2}}=\sum_{n=0}^{\infty}(-1)^{n} \frac{I_{n}}{n !} t^{n} .
$$


Одним из применений этой теоремы является следствие 5.

Теперь свяжем $I_{-1}$ с константой Эйлера $\gamma$, которая определяется как предел

$$
\gamma=\lim _{n \rightarrow \infty}\left(1+\frac{1}{2}+\cdots+\frac{1}{n}-\ln n\right)
$$

Если рассматривать $\gamma$ как " $\zeta(1)$ ", то формулы

$$
I_{2}=\frac{9}{2} \zeta(4), \quad I_{1}=2 \zeta(3), \quad I_{0}=\zeta(2)
$$

дают основание полагать, что $I_{-1}$ содержит $\gamma$. Это предположение также следует из сходства между двойным интегралом (7) для $I_{-1}$ и двойным интегралом для константы Эйлера [8], [9]

$$
\gamma=\iint_{S} \frac{1-X}{(1-X Y)(-\ln X Y)} d X d Y
$$

Формула (8) приводит к другой формуле, связывающей $I_{-1}$ и $\gamma$. Именно, если $t=n$ - положительное целое число, то $\left(\begin{array}{c}2 t \\ t\end{array}\right)$ - центральный биномиальный коэффициент $\left(\begin{array}{c}2 n \\ n\end{array}\right)$, фигурирующий в формулах для константы Эйлера

$$
\left(\begin{array}{c}
2 n \\
n
\end{array}\right) \gamma=A_{n}-L_{n}+\iint_{S} \frac{(X(1-X) Y(1-Y))^{n}}{(1-X Y)(-\ln X Y)} d X d Y, \quad n \geqslant 1,
$$

и

$$
\gamma=\frac{A_{n}-L_{n}}{\left(\begin{array}{c}
2 n \\
n
\end{array}\right)}+O\left(\frac{1}{2^{6 n} \sqrt{n}}\right), \quad n \rightarrow \infty,
$$

где $A_{n}$ - некоторое рациональное число и $L_{n}-$ частная линейная форма в логариф$\max [8]$.

Если произвести в (10) замену переменных $X=x, Y=(1-y) / x$, то мы получим интеграл по треугольнику $T$ для константы Эйлера,

$$
\gamma=\iint_{T} \frac{1-x}{x y(-\ln (1-y))} d x d y
$$

аналогичный интегралу по треугольнику (7) для $I_{-1}$.

Найдем аналог для $\gamma$ первого интеграла в формуле (8) для $I_{-1}$, который содержит обобщенный биномиальный коэффициент $\left(\begin{array}{c}2 t \\ t\end{array}\right)$. (Существуют классические аналоги для $\gamma$ второго интеграла, содержащие логарифмы.)

ПреДЛОЖЕНИЕ 2. Справедлива следующая формула для константы Эйлера:

$$
\gamma=\int_{0}^{\infty} \sum_{k=2}^{\infty} \frac{1}{k^{2}\left(\begin{array}{c}
t+k \\
k
\end{array}\right)} d t
$$

В качестве примера заметим, что если проинтегрировать почленно и пропотенциировать полученный ряд, то мы получим бесконечное произведение Сера для е ${ }^{\gamma}[10]$ (заново полученное в [11], [12]):

$$
e^{\gamma}=\prod_{k=2}^{\infty}\left(\prod_{j=1}^{k} j^{(-1)^{j}\left(\begin{array}{c}
k-1 \\
j-1
\end{array}\right)}\right)^{1 / k}=\left(\frac{2}{1}\right)^{1 / 2}\left(\frac{2^{2}}{1 \cdot 3}\right)^{1 / 3}\left(\frac{2^{3} \cdot 4}{1 \cdot 3^{3}}\right)^{1 / 4}\left(\frac{2^{4} \cdot 4^{4}}{1 \cdot 3^{6} \cdot 5}\right)^{1 / 5} \cdots
$$


Оставшаяся часть статьи строится следующим образом. В пп. 2 и 3 получены соответственно неасимптотические и асимптотические результаты для $I_{n}$ при $n \geqslant 0$. Примеры применения этих результатов для кратных дзета значений рассматриваются в п. 4, а в п. 5 доказываются формулы для $I_{-1}$ и $\gamma$. Заключительный пункт посвящен обобщению $I_{n}$ на случай интегралов по многомерным аналогам треугольника $T$; один из которых - повторный интеграл Чена (Дринфилда-Концевича), см. замечание 3. В качестве примера рассматривается соотношение между некоторыми кратными полилогарифмическими значениями и кратными дзета значениями (следствие 7).

2. Интеграл $I_{n}$ для $n \geqslant 0$. Докажем неасимптотические результаты для $I_{0}, I_{1}$, $I_{2}, \ldots$, сформулированные во введении. Для удобства читателя мы повторяем здесь формулировки доказываемых результатов (лемма 1, теорема 1, следствие 1).

Лемма 1. Если $k \geqslant 0$ u $l \geqslant 0-$ целье, то

$$
I_{k, l}:=\iint_{T} \frac{(-\ln x)^{k}(-\ln y)^{l}}{x y} d x d y=k ! l ! \zeta\left(l+2,\{1\}_{k}\right) .
$$

Кроме того, если $l \geqslant 1$, то

$$
J_{k, l}:=\int_{0}^{1} \frac{(-\ln (1-x))^{k}}{1-x}(-\ln x)^{l} d x=k ! l ! \zeta\left(l+1,\{1\}_{k}\right) .
$$

ДокАЗАТЕЛЬСТво. Имеем

$$
I_{k, l}=\int_{0}^{1} \frac{(-\ln x)^{k}}{x} \int_{1-x}^{1} \frac{(-\ln y)^{l}}{y} d y d x=\int_{0}^{1} \frac{(-\ln x)^{k}}{x} \cdot \frac{(-\ln (1-x))^{l+1}}{l+1} d x .
$$

Заменяя $x$ на $1-x$, получим

$$
I_{k, l}=\frac{J_{k, l+1}}{l+1} .
$$

Таким образом, (4) следует из (5). Чтобы доказать (5), умножим формулу [7; раздел 1]

$$
(-\ln (1-x))^{k}=k ! \sum_{n_{1}>n_{2}>\cdots>n_{k}>0} \frac{x^{n_{1}}}{n_{1} \cdots n_{k}}
$$

на $(1-x)^{-1}=1+x+x^{2}+\cdots$ и подставим полученный ряд

$$
\frac{(-\ln (1-x))^{k}}{1-x}=k ! \sum_{m \geqslant n_{1}>n_{2}>\cdots>n_{k}>0} \frac{x^{m}}{n_{1} \cdots n_{k}}
$$

в интеграл (5) для $J_{k, l}$. Затем, интегрируя почленно и учитывая, что

$$
\int_{0}^{1} x^{m}(-\ln x)^{l} d x=\frac{l !}{(m+1)^{l+1}}
$$

получим

$$
J_{k, l}=k ! l ! \sum_{m>n_{1}>n_{2}>\cdots>n_{k}>0} \frac{1}{m^{l+1} n_{1} \cdots n_{k}}=k ! l ! \zeta\left(l+1,\{1\}_{k}\right),
$$

что доказывает утверждение леммы. 
Докажем теорему 1 в следующей формулировке.

ТеОРема 1. Если $n \geqslant 0$, то $I_{n}$ можно представить следующими способами:

(i) через кратные дзета значения в виде

$$
I_{n}=n ! \sum_{k=0}^{n} \zeta\left(n-k+2,\{1\}_{k}\right)
$$

(ii) через обычные дзета значения в виде

$$
I_{n}=\sum_{k=0}^{n}\left(\begin{array}{l}
n \\
k
\end{array}\right) \frac{J_{k, n-k+1}}{n-k+1},
$$

где интеграл $J_{k, n-k+1}$, определенный в (5), задается формулой [5]

$$
J_{k, l}=k ! l ! \sum_{p=1}^{l} \frac{(-1)^{p+1}}{p !} \sum_{t_{i}} \frac{\zeta\left(t_{1}\right) \cdots \zeta\left(t_{p}\right)}{t_{1} \cdots t_{p}} \sum_{l_{i}}\left(\begin{array}{c}
t_{1} \\
l_{1}
\end{array}\right) \cdots\left(\begin{array}{c}
t_{p} \\
l_{p}
\end{array}\right),
$$

сумма по $t_{i}$ берется по всем множествам иельх чисел $\left\{t_{1}, \ldots, t_{p}\right\}$ таких, что

$$
t_{i}>1, \quad \sum_{i=1}^{p} t_{i}=k+l+1,
$$

и сумма по $l_{i}$ берется по всем множествам иелых чисел $\left\{l_{1}, \ldots, l_{p}\right\}$ таких, что

$$
0<l_{i}<t_{i}, \quad \sum_{i=1}^{p} l_{i}=l .
$$

ДоказАтельство. Разлагая $(-\ln x y)^{n}=(-\ln x-\ln y)^{n}$ в определении (1) интеграла $I_{n}$ и применяя (4), получим (12). Следовательно, применяя (5), получим формулу (13). Наконец, заметим, что оценка (14) интеграла (5) для $J_{k, l}$ доказана в [5].

СлеДСТВИЕ 1. Для $n=0,1,2$ интеграл $I_{n}$ является рационалъным кратным числа $\zeta(n+2)$.

Мы приведем два доказательства этого утверждения. Первое доказательство короткое, но оно использует теорему 1, (ii), доказательство которой дано в [5]. Второе доказательство длиннее, но оно самодостаточное (за исключением доказательства формулы Эйлера): оно использует теорему 1, (i) и следствие 3, доказательства которых не зависят от результатов, полученных в других статьях.

ДокАЗАТЕЛЬСтво 1. При $n=0,1,2$ из формул (13) и (14) следует, что

$$
I_{0}=J_{0,1}=\zeta(2), \quad I_{1}=\frac{1}{2} J_{0,2}+J_{1,1}=2 \zeta(3), \quad I_{2}=\frac{1}{3} J_{0,3}+\frac{1}{2} J_{1,2}+J_{2,1}=\frac{9}{4} \zeta(4) .
$$

ДокАЗАтЕЛЬСтво 2. Во введении было показано, что $I_{0}=\zeta(2)$. Применяя тот же метод и формулу $\int_{0}^{1} x^{k-1}(-\ln x) d x=k^{-2}$, получим

$$
I_{1}=2 \iint_{T} \frac{-\ln x}{x y} d x d y=2 \int_{0}^{1} \frac{\ln (1-x)}{x} \ln x d x=2 \sum_{k=1}^{\infty} \frac{1}{k} \int_{0}^{1} x^{k-1}(-\ln x) d x=2 \zeta(3) .
$$


С другой стороны, $I_{0}=\zeta(2)$ и $I_{1}=\zeta(3)+\zeta(2,1)$ в силу теоремы $1,(\mathrm{i})$ и $\zeta(2,1)=$ $\zeta(3)$ в силу следствия 3.

Чтобы доказать, что $I_{2}=(9 / 2) \zeta(4)$, достаточно в силу теоремы $1,(\mathrm{i})$ и следствия 3 применить формулу Эйлера $\zeta(3,1)=\zeta(4) / 4$. (Для доказательства последней положим $n=3$ в его уравнении (9.5) в [13; с. 252].)

ТеОРема 2. Если числа $\pi, \zeta(3), \zeta(5), \zeta(7), \zeta(9), \ldots$ алгебраически независимы над полем рачиональных чисел, то $I_{n}$ не является рачиональным кратным числа $\zeta(n+2)$ nрu $n>2$.

ДоказАтельство. Сначала рассмотрим случай $n=3 m-2$ при $m>1$. Интеграл $I_{n}$ равен линейной комбинации (13) интегралов $J_{k, l}$ с положительными коэффициентами. Каждый $J_{k, l}$ равен полиному (14) от многих переменных относительно обычных дзета значений. Теперь в (14) моном $\zeta(3)^{m}$ появляется только при $p=m$, и тогда его коэффициент не равен нулю и имеет знак $(-1)^{m+1}$. Следовательно, в выражении для $I_{n}$ коэффициент $\zeta(3)^{m}$ не равен нулю. Отсюда следует, что по условию $I_{n}$ не может быть рациональным кратным числа $\zeta(n+2)=\zeta(3 m)$.

Случаи $n=3 m+3$ и $n=3 m+5$ при $m>0$ аналогичны: рассматриваются соответственно мономы $\zeta(3)^{m} \zeta(5)$ и $\zeta(3)^{m} \zeta(7)$. Оставшиеся случаи $n=3$ и $n=5$ доказываются прямыми вычислениями. Таким образом, теорема доказана.

Перейдем к доказательству теоремы 5. Для удобства читателя напомним ее формулировку.

TеOPEMA 5. Eсли $0<|t|<1$, mo

$$
\frac{1}{t^{2}}\left(1-\frac{1}{\left(\begin{array}{c}
2 t \\
t
\end{array}\right)}\right)=\sum_{n=0}^{\infty}(-1)^{n} \frac{I_{n}}{n !} t^{n} .
$$

ДокАзАТЕЛЬСтво. Производящая функция

$$
\sum_{k, l \geqslant 0} x^{k+1} y^{l+1} \zeta\left(l+2,\{1\}_{k}\right)=1-\frac{\Gamma(1-x) \Gamma(1-y)}{\Gamma(1-x-y)}
$$

(ср. с (19)) выводится в [6]. Если $x=y=-t$, то ряд сходится при $|t|<1$. Полагая $k+l=n$, получим

$$
t^{2} \sum_{n=0}^{\infty}(-1)^{n} t^{n} \sum_{k=0}^{n} \zeta\left(n-k+2,\{1\}_{k}\right)=1-\frac{1}{\left(\begin{array}{c}
2 t \\
t
\end{array}\right)} .
$$

Применяя (2), завершаем доказательство теоремы.

3. Асимптотическое разложение интеграла $I_{n}$. Применяя теорему 5 и следующую лемму, оценим интеграл $I_{n}$ при больших $n$.

ЛЕмма 2. Предположим, что функиия $f(z)$ мероморфна в комплексной плоскости и имеет только простые полюса $z_{1}, z_{2}, \ldots$ с вычетами $r_{1}, r_{2}, \ldots$ соответственно. Если $0<\left|z_{1}\right| \leqslant\left|z_{2}\right| \leqslant \cdots$, то коэфбициенты ряда Тейлора

$$
f(z)=\sum_{n=0}^{\infty} a_{n} z^{n}
$$


имеют асимптотическое разложение

$$
a_{n} \approx-\frac{r_{1}}{z_{1}^{n+1}}-\frac{r_{2}}{z_{2}^{n+1}}-\cdots, \quad n \rightarrow \infty .
$$

ЗАмечАНИЕ 1. Напомним [14; раздел 1.5], что из последней формулы следует, что, при любом фиксированном положительном целом числе $k$

$$
a_{n}=-\frac{r_{1}}{z_{1}^{n+1}}-\cdots-\frac{r_{k}}{z_{k}^{n+1}}+O\left(\frac{r_{k+1}}{z_{k+1}^{n+1}}\right), \quad n \rightarrow \infty .
$$

ДокАЗАТЕЛЬСтво ЛЕммы 2. Мероморфная функция имеет только конечное число полюсов в любой ограниченной области, таким образом, для любого $k \geqslant 1$ существует $l>k$ такой, что $\left|z_{l}\right|<\left|z_{l+1}\right|$. Заметим, что единственными особенностями функции

$$
f(z)-\sum_{j=1}^{l} \frac{r_{j}}{z-z_{j}}=\sum_{n=0}^{\infty}\left(a_{n}+\frac{r_{1}}{z_{1}^{n+1}}+\cdots+\frac{r_{l}}{z_{l}^{n+1}}\right) z^{n}
$$

являются $z_{l+1}, z_{l+2}, \ldots$. Отсюда и из неравенств $0<\left|z_{1}\right| \leqslant\left|z_{2}\right| \leqslant \cdots$ следует, что радиус сходимости ряда (18) равен $\left|z_{l+1}\right|$. Так как $\left|z_{l}\right|<\left|z_{l+1}\right|$, можно подставить $z=z_{l}$ в эти ряды. Следовательно,

$$
\lim _{n \rightarrow \infty}\left(a_{n}+\frac{r_{1}}{z_{1}^{n+1}}+\cdots+\frac{r_{k}}{z_{k}^{n+1}}+\frac{r_{k+1}}{z_{k+1}^{n+1}}+\cdots+\frac{r_{l}}{z_{l}^{n+1}}\right) z_{l}^{n}=0 .
$$

Так как $\left|z_{k+1}\right| \leqslant\left|z_{k+2}\right| \leqslant \cdots \leqslant\left|z_{l}\right|$, в пределе получаем асимптотическую формулу (17). Лемма доказана.

ТЕОРема 3. Соотношение $I_{n} \sim 2 n$ ! выполняется при $n$, стремящемся $к$ бесконечности. Более точно, имеет место следующее асимптотическое разложение:

$$
\frac{I_{n}}{n !} \approx 2+\frac{6}{2^{n+2}}+\frac{20}{3^{n+2}}+\frac{70}{4^{n+2}}+\cdots, \quad n \rightarrow \infty,
$$

где числитель в $k$-м члене равен $\left(\begin{array}{c}2 k \\ k\end{array}\right)$ при $k=1,2, \ldots$.

ДокАзАтЕЛЬСтво. Обозначим функцию в левой части (9) через $f(t)$. Кроме устранимой особенности в точке $t=0$, все особенности функции $f(t)$ являются простыми полюсами в точках $t=-1,-2, \ldots$. Вычисления показывают, что вычет в точке $t=-k$ равен

$$
\operatorname{Res}(f ;-k)=\operatorname{Res}\left(\frac{1}{t^{2}}\left(1-\frac{\Gamma(t+1)^{2}}{\Gamma(2 t+1)}\right) ;-k\right)=-\frac{1}{k^{2}} \lim _{t \rightarrow-k}(t+k) \frac{\Gamma(t+1)^{2}}{\Gamma(2 t+1)}=\frac{1}{k}\left(\begin{array}{c}
2 k \\
k
\end{array}\right)
$$

при $k=1,2, \ldots$. Применяя теорему 5 и лемму 2 , получим второе утверждение теоремы (из которого следует первое утверждение).

СлеДСТВИЕ 5. Ряд

$$
\sum_{n=0}^{\infty}(-1)^{n} \frac{I_{n}}{n !}
$$

расходится, но являтся суммируемым по Абелю, и его сумма равна $1 / 2$.

Доказательство. Расходимость следует из (6). Полагая, что $t \rightarrow 1^{-}$в (15), получаем требуемое суммирование по Абелю. 
4. Примеры в случае кратных дзета значений. Применяя результаты, полученные для $I_{n}$, рассмотрим кратные дзета значения вида $\zeta\left(m,\{1\}_{k}\right)$.

СлЕДСТвиЕ 2. Если $m \geqslant 2 u k \geqslant 0$, то кратное дзета значение $\zeta\left(m,\{1\}_{k}\right)$ можно представить в явном виде как полином от многих переменных с рациональными коэффичиентами относительно обычных дзета значений $\zeta(2), \zeta(3), \ldots, \zeta(m+k)$.

ДоКАЗАТЕЛЬСТво. Положим $l=m-1$ в (5) и (14).

ЗАмЕчАНИЕ 2. Этот результат, включая полиномиальную формулу (по крайней мере, неявную), был впервые получен в [6] с помощью производящей функции (см. [6] касательно равносильности с (16))

$$
\sum_{k, l \geqslant 0} x^{k+1} y^{l+1} \zeta\left(l+2,\{1\}_{k}\right)=1-\exp \left(\sum_{n=2}^{\infty} \frac{x^{n}+y^{n}-(x+y)^{n}}{n} \zeta(n)\right) .
$$

СлЕДСТВИЕ 3. Если $k \geqslant 0 u l \geqslant 0, m o$

$$
\zeta\left(k+2,\{1\}_{l}\right)=\zeta\left(l+2,\{1\}_{k}\right) .
$$

ДокАзАтельство. После замены переменных $x, y \rightarrow y, x$ в интеграле (4) из симметрии треугольника $T$ следует, что $I_{k, l}=I_{l, k}$. Применяя лемму 1 , получаем искомый результат.

СЛЕДСтвиЕ 4. Среднее кратных дзета значений $\zeta(m), \zeta(m-1,1), \ldots, \zeta\left(2,\{1\}_{m-2}\right)$ асимптотически стремится $\kappa 2 / m$ при $m$, стремящемся $\kappa$ бесконечности. Действительно, имеет место следующее асимптотическое разложение:

$$
\sum_{k=0}^{m-2} \zeta\left(m-k,\{1\}_{k}\right) \approx 2+\frac{6}{2^{m}}+\frac{20}{3^{m}}+\frac{70}{4^{m}}+\cdots, \quad m \rightarrow \infty .
$$

ДокАЗАТЕЛЬство. Полагая $m=n+2$ в теоремах 1 и 3 , получаем искомое разложение. Отсюда, искомое среднее асимптотически стремится к $2 /(m-1) \sim 2 / m$ при $m$, стремящемся к бесконечности.

5. Интеграл $I_{-1}$ и константа Эйлера. Докажем утверждения, сформулированные для $I_{-1}$ и $\gamma$ во введении. Как обычно, для удобства читателя повторим здесь их формулировки.

ПрЕДЛОЖЕНИЕ 1. Имеют место следующие формулы, выражающие двойной интеграл $I_{-1}$ через простые интегралы:

$$
I_{-1}=\int_{0}^{\infty}\left(1-\frac{1}{\left(\begin{array}{c}
2 t \\
t
\end{array}\right)}\right) \frac{d t}{t^{2}}=\int_{0}^{1} \ln \left(1+\frac{\ln (1-x)}{\ln x}\right) \frac{d x}{x} .
$$

ДокАЗАТЕЛЬСтво. В (7), делая замену

$$
-\frac{1}{\ln x y}=\int_{0}^{\infty}(x y)^{t} d t
$$


и меняя порядок интегрирования, получим

$$
\begin{aligned}
I_{-1} & =\int_{0}^{\infty} \int_{0}^{1} \int_{1-x}^{1}(x y)^{t-1} d y d x d t=\int_{0}^{\infty} \int_{0}^{1}\left(x^{t-1}-x^{t-1}(1-x)^{t}\right) d x \frac{d t}{t} \\
& =\int_{0}^{\infty}\left(\frac{1}{t}-\frac{\Gamma(t) \Gamma(t+1)}{\Gamma(2 t+1)}\right) \frac{d t}{t},
\end{aligned}
$$

применяя интеграл Эйлера для бета функции. После замены $\Gamma(t)$ на $t^{-1} \Gamma(t+1)$, получим первое равенство. Интегрируя по $y$ в (7), видим, что второй интеграл также равен $I_{-1}$.

Теорема 4. Если li - логарифмический интеграл, то

$$
I_{-1}=\sum_{n=0}^{\infty}(-1)^{n} \frac{I_{n}}{(n+1) !}+\int_{0}^{1} \frac{\operatorname{li}\left(x-x^{2}\right)}{x} d x+1 .
$$

ДокАЗАТЕЛЬСтво. Подставим

$$
-\frac{1}{\ln x y}=\int_{0}^{1}(x y)^{t} d t-\frac{x y}{\ln x y}
$$

в (7). Следуя доказательству предложения 1, получим

$$
I_{-1}=\int_{0}^{1}\left(1-\frac{1}{\left(\begin{array}{c}
2 t \\
t
\end{array}\right)}\right) \frac{d t}{t^{2}}-\iint_{T} \frac{d x d y}{\ln x y} .
$$

Подставляя ряд (15) в первый интеграл и интегрируя почленно, получим ряд в искомой формуле. Полагая $y=u / x$ во втором интеграле, получим

$$
\iint_{T} \frac{d x d y}{\ln x y}=\int_{0}^{1} \frac{1}{x} \int_{x-x^{2}}^{x} \frac{d u}{\ln u} d x=\int_{0}^{1} \frac{\operatorname{li}(x)-\operatorname{li}\left(x-x^{2}\right)}{x} d x,
$$

и, проводя следующие вычисления (см. [15; раздел 6.212]), завершим доказательство:

$$
\int_{0}^{1} \frac{\operatorname{li}(x)}{x} d x=\lim _{q \rightarrow 0} \int_{0}^{1} \frac{\operatorname{li}(x)}{x^{q+1}} d x=\lim _{q \rightarrow 0} \frac{\ln (1-q)}{q}=-1 .
$$

ПредложениЕ 2. Имеет место следующая формула для константы Эйлера:

$$
\gamma=\int_{0}^{\infty} \sum_{k=2}^{\infty} \frac{1}{k^{2}\left(\begin{array}{c}
t+k \\
k
\end{array}\right)} d t
$$

ДокАЗАтеЛЬСтво. В (20) заменим $x y$ на $1-y$. Подставляя результат в (11) и меняя порядок интегрирования, получим

$$
\begin{aligned}
\gamma & =\int_{0}^{\infty} \int_{0}^{1} \int_{1-y}^{1} \frac{1-x}{x y}(1-y)^{t} d x d y d t=\int_{0}^{\infty} \int_{0}^{1} \frac{-\ln (1-y)-y}{y}(1-y)^{t} d y d t \\
& =\int_{0}^{\infty} \sum_{k=2}^{\infty} \frac{1}{k} \int_{0}^{1} y^{k-1}(1-y)^{t} d y d t .
\end{aligned}
$$

Следуя доказательству предложения 1, приходим к искомой формуле. 
6. Интегралы по многомерным аналогам треугольника T. Существует несколько путей обобщения треугольника $T$ и интеграла $I_{n}$. Простейшим обобщением $T$ является многогранник

$$
V_{m}:=\left\{\left(x_{1}, x_{2}, \ldots, x_{m}\right) \in[0,1]^{m} \mid x_{1}+x_{j} \geqslant 1, j=2, \ldots, m\right\} .
$$

ТЕОРема 6. При $m \geqslant 2 u n \geqslant 0$ интеграл

$$
K_{m, n}:=\int \cdots \int_{V_{m}} \frac{\left(-\ln \left(x_{1} x_{2} \cdots x_{m}\right)\right)^{n}}{x_{1} x_{2} \cdots x_{m}} d x_{1} d x_{2} \cdots d x_{m}
$$

равен целой линейной комбинации кратных дзета значений с весом $m+n, a$ именно,

$$
K_{m, n}=n ! \sum_{\substack{k_{1} \geqslant 0, \ldots, k_{m} \geqslant 0 \\ k_{1}+\cdots+k_{m}=n}} \frac{\left(k_{2}+\cdots+k_{m}+m-1\right) !}{\left(k_{2}+1\right) ! \cdots\left(k_{m}+1\right) !} \zeta\left(k_{2}+\cdots+k_{m}+m,\{1\}_{k_{1}}\right) .
$$

Он также равен полиному от многих переменных с рачиональными коэфбициентами относительно значений дзета функции Римана в целых точках.

ДоказАтельство. Разлагая $\left(-\ln \left(x_{1} x_{2} \cdots x_{m}\right)\right)^{n}=\left(-\ln x_{1}-\ln x_{2}-\cdots-\ln x_{m}\right)^{n}$, получим

$$
\begin{aligned}
K_{m, n}= & \sum_{\substack{k_{1} \geqslant 0, \ldots, k_{m} \geqslant 0 \\
k_{1}+\cdots+k_{m}=n}} \frac{n !}{k_{1} ! k_{2} ! \cdots k_{m} !} \\
& \quad \times \int_{0}^{1} \frac{\left(-\ln x_{1}\right)^{k_{1}}}{x_{1}}\left(\int_{1-x_{1}}^{1} \frac{\left(-\ln x_{2}\right)^{k_{2}}}{x_{2}} d x_{2} \cdots \int_{1-x_{1}}^{1} \frac{\left(-\ln x_{m}\right)^{k_{m}}}{x_{m}} d x_{m}\right) d x_{1} .
\end{aligned}
$$

Taк как

$$
\int_{1-x_{1}}^{1} \frac{(-\ln x)^{k}}{x} d x=\frac{\left(-\ln \left(1-x_{1}\right)\right)^{k+1}}{k+1}
$$

мы имеем

$$
\begin{aligned}
K_{m, n}= & \sum_{\substack{k_{1} \geqslant 0, \ldots, k_{m} \geqslant 0 \\
k_{1}+\cdots+k_{m}=n}} \frac{n !}{k_{1} !\left(k_{2}+1\right) ! \cdots\left(k_{m}+1\right) !} \\
& \int_{0}^{1} \frac{\left(-\ln x_{1}\right)^{k_{1}}}{x_{1}}\left(-\ln \left(1-x_{1}\right)\right)^{k_{2}+\cdots+k_{m}+m-1} d x_{1} .
\end{aligned}
$$

Вычисляя последний интеграл с помощью формул (5) и (14), приходим к утверждению теоремы.

В случае $m=2$ многогранник $V_{2}$ является треугольником $T$, интеграл $K_{2, n}$ является треугольным интегралом $I_{n}$ и теорема 6 сводится к теореме 1 . В частности, формула (21) для $K_{m, n}$ является весовой версией формулы (2) для $I_{n}$.

СлЕДСТВИЕ 6 . Если $m \geqslant 2, m o$

$$
K_{m, 0}=(m-1) ! \zeta(m) .
$$

ДокАЗАтЕЛЬСтво. Полагая $n=0$ в теореме 6 , получим $k_{1}=k_{2}=\cdots=k_{m}=0$ в (21). 
Существует другое, более естественное доказательство следствия 6 , которое не использует теорему 6.

ВТОРОЕ ДОКАЗАТЕЛЬСТВо СЛЕДСТВИЯ 6. Воспользуемся представлением

$$
\zeta(m)=K_{m}^{\prime}:=\int \cdots \int_{[0,1]^{m}} \frac{d x_{1} \cdots d x_{m}}{1-x_{1} \cdots x_{m}} .
$$

(Чтобы доказать эту формулу, разложим подынтегральное выражение в геометрический ряд и проинтегрируем почленно.) Проведем замену переменных

$$
x_{1}=y_{m}, \quad x_{2}=\frac{y_{m-1}}{y_{m}}, \quad x_{3}=\frac{y_{m-2}}{y_{m-1}}, \quad \ldots, \quad x_{m-1}=\frac{y_{2}}{y_{3}}, \quad x_{m}=\frac{1-y_{1}}{y_{2}} .
$$

(При $m=2$ сравним это выражение с преобразованием интеграла $I_{0}$ в интеграл Бейкерса для $\zeta(2)$ во введении.) Получим

$$
\zeta(m)=\int \cdots \int \frac{d y_{1} \cdots d y_{m}}{y_{1} \cdots y_{m}},
$$

где интеграл берется по многограннику, определенному как $1 \geqslant y_{m} \geqslant y_{m-1} \geqslant \cdots \geqslant$ $y_{2} \geqslant 0, y_{2}+y_{1} \geqslant 1, y_{1} \leqslant 1$. В силу симметрии можно поменять между собой переменные $y_{i}$ и $y_{j}$ при $i>j>1$. Применяя все перестановки переменных $y_{2}, \ldots, y_{m}$, получим

$$
(m-1) ! \zeta(m)=\int \cdots \int_{V_{m}^{\prime}} \frac{d y_{1} \cdots d y_{m}}{y_{1} \cdots y_{m}},
$$

где интеграл берется по

$$
V_{m}^{\prime}:=\left\{\left(y_{1}, y_{2}, \ldots, y_{m}\right) \in[0,1]^{m} \mid y_{1}+y_{j} \geqslant 1, j=2, \ldots, m\right\} .
$$

Это и есть интеграл $K_{m, 0}$ и, таким образом, второе доказательство завершено.

ЗАмечаниЕ 3. Интеграл $K_{m}^{\prime}$ равносилен интегралу Чена (Дринфилда-Концевича) [7; раздел 1]

$$
\int \cdots \int \frac{d Y_{1} d Y_{2} \cdots d Y_{m}}{\left(1-Y_{1}\right) Y_{2} \cdots Y_{m}}
$$

по многограннику $1 \geqslant Y_{m} \geqslant \cdots \geqslant Y_{1} \geqslant 0$ : положим $y_{1}=1-Y_{1}$ и $y_{j}=Y_{j}$ для $j=2, \ldots, m$.

Если переписать (21) в виде

$$
K_{m, n}=n ! \sum_{p=0}^{n} a_{m, p} \zeta\left(m+p,\{1\}_{n-p}\right),
$$

где $a_{m, p}$ обозначает сумму коэффициентов полинома

$$
a_{m, p}:=\sum_{\substack{k_{2} \geqslant 0, \ldots, k_{m} \geqslant 0 \\ k_{2}+\cdots+k_{m}=p}} \frac{\left(k_{2}+\cdots+k_{m}+m-1\right) !}{\left(k_{2}+1\right) ! \cdots\left(k_{m}+1\right) !},
$$

то для любого фиксированного $m \geqslant 2$ можно получить замкнутое выражение для $a_{m, p}$. Например,

$$
a_{2, p}=1, \quad a_{3, p}=4 \cdot 2^{p}-2, \quad a_{4, p}=27 \cdot 3^{p}-24 \cdot 2^{p}+3 .
$$

В общем случае имеем следующее утверждение. 
ПреДЛОЖЕНИЕ 3. Выберем $m \geqslant 2 u p \geqslant 0$. Тогда челые числа $a_{m, p}, a_{m-1, p+1}, \ldots$, $a_{2, p+m-2}$ удовлетворяют рекуррентному соотношению

$$
\sum_{t=0}^{m-2}\left(\begin{array}{c}
m-1 \\
t
\end{array}\right) a_{m-t, p+t}=(m-1)^{m+p-1} .
$$

ДокАЗАтЕльство. Сначала заметим, что если ввести обозначение

$$
S_{m, p}:=\left\{\left(k_{2}, \ldots, k_{m}\right) \in \mathbb{Z}^{m-1} \mid k_{j} \geqslant-1, j=2, \ldots, m ; k_{2}+\cdots+k_{m}=p\right\},
$$

Tо

$$
\sum_{S_{m, p}} \frac{\left(k_{2}+\cdots+k_{m}+m-1\right) !}{\left(k_{2}+1\right) ! \cdots\left(k_{m}+1\right) !}=\sum_{\substack{l_{2} \geqslant 0, \ldots, l_{m} \geqslant 0 \\ l_{2}+\cdots+l_{m}=p+m-1}} \frac{\left(l_{2}+\cdots+l_{m}\right) !}{l_{2} ! \cdots l_{m} !}=(m-1)^{p+m-1} .
$$

Теперь заметим, что если $S_{m, p, t}$ - подмножество множества $S_{m, p}$, состоящего из тех $(m-1)$-наборов $\left(k_{2}, \ldots, k_{m}\right)$, в которых в точности $t$ чисел среди $k_{j}$ равны -1 , то

$$
\begin{aligned}
\sum_{S_{m, p, t}} \frac{\left(k_{2}+\cdots+k_{m}+m-1\right) !}{\left(k_{2}+1\right) ! \cdots\left(k_{m}+1\right) !} & =\left(\begin{array}{c}
m-1 \\
t
\end{array}\right) \sum_{\substack{l_{2} \geqslant 0, \ldots, l_{m} \geqslant 0 \\
l_{2}+\cdots+l_{m-t}=p+t}} \frac{\left(l_{2}+\cdots+l_{m}+m-1-t\right) !}{\left(l_{2}+1\right) ! \cdots\left(l_{m}+1\right) !} \\
& =\left(\begin{array}{c}
m-1 \\
t
\end{array}\right) a_{m-t, p+t .} .
\end{aligned}
$$

Наконец, из того, что $S_{m, p}$ - объединение непересекающихся множеств,

$$
S_{m, p}=\bigcup_{t=0}^{m-2} S_{m, p, t}
$$

следует утверждение предложения.

Другим обобщением треугольника $T$ является многогранник

$$
W_{m}:=\left\{\left(x_{1}, \ldots, x_{m}\right) \in[0,1]^{m} \mid x_{i}+x_{j} \geqslant 1,1 \leqslant i<j \leqslant m\right\} .
$$

Заметим, что он симметричен по всем переменным в отличие от $V_{m}$.

Сначала мы обобщим треугольный интеграл $I_{0}$ на интеграл по $W_{m}$, а затем продолжим обобщение интегралов $I_{n}$ на $W_{m}$ для всех $n \geqslant 0$.

Напомним, что для всех комплексных $s$ и всех $z$ таких, что $|z|<1$, полилогарuфм $\mathrm{Li}_{s}(z)$ определяется сходящимся рядом

$$
\operatorname{Li}_{s}(z):=\sum_{r=1}^{\infty} \frac{z^{r}}{r^{s}}
$$

ТеОРема 7. Если $m \geqslant 2$, то интеграл

$$
L_{m}:=\int \cdots \int_{W_{m}} \frac{d x_{1} \cdots d x_{m}}{x_{1} \cdots x_{m}}
$$

равен полиному от многих переменных с рациональными коэффициентами относительно значений $\ln 2, \zeta(m) u \operatorname{Li}_{s}(1 / 2)$ при $s=2,3, \ldots, m$, именно,

$$
L_{m}=m ! \zeta(m)-(m-1) \ln ^{m} 2-m ! \sum_{p=0}^{m-2} \frac{\ln ^{p} 2}{p !} \operatorname{Li}_{m-p}\left(\frac{1}{2}\right) .
$$


ДокАЗАТЕЛЬСТво. Из симметрии $W_{m}$ следует, что

$$
L_{m}=m ! \int \cdots \int_{W_{m}^{\prime}} \frac{d x_{1} \cdots d x_{m}}{x_{1} \cdots x_{m}}
$$

где $W_{m}^{\prime}$ - многогранник, заданный неравенствами $0 \leqslant x_{1} \leqslant x_{2} \leqslant \cdots \leqslant x_{m} \leqslant 1$ и $x_{1}+x_{2} \geqslant 1$. Последовательно интегрируя по $x_{m}, x_{m-1}, \ldots, x_{2}$ и полагая, что $x=x_{1}$, $y=x_{2}$, получим

$$
L_{m}=m(m-1) \iint_{H} \frac{(-\ln y)^{m-2}}{x y} d x d y,
$$

где $H$ - треугольник, заданный неравенствами $0 \leqslant x \leqslant y \leqslant 1$ и $x+y \geqslant 1$. (Таким образом, $H$ является верхней половиной треугольника $T$, разделенного пополам линией $y=x$.) Так как $H$ также определяется неравенствами $1 / 2 \leqslant y \leqslant 1$ и $1-y \leqslant x \leqslant y$, мы видим, что

$$
\begin{aligned}
L_{m} & =m(m-1) \int_{1 / 2}^{1} \frac{(-\ln y)^{m-2}(\ln y-\ln (1-y))}{y} d y \\
& =(m-1)\left(-(\ln 2)^{m}+m \int_{1 / 2}^{1} \frac{(-\ln y)^{m-2}(-\ln (1-y))}{y} d y\right) .
\end{aligned}
$$

Разложение в ряд

$$
\frac{-\ln (1-y)}{y}=\sum_{r=1}^{\infty} \frac{y^{r-1}}{r}
$$

приводит к равенству

$$
\int_{1 / 2}^{1} \frac{(-\ln y)^{m-2}(-\ln (1-y))}{y} d y=\sum_{r=1}^{\infty} \frac{1}{r} \int_{1 / 2}^{1} y^{r-1}(-\ln y)^{m-2} d y .
$$

Теперь рассмотрим тождество

$$
\int_{1 / 2}^{1} y^{t+r-1} d y=\frac{1}{t+r}-\frac{1}{(t+r) 2^{t+r}} .
$$

Если его продифференцировать $l$ раз по $t$ и затем положить $t=0$, то мы получим

$$
\int_{1 / 2}^{1} y^{r-1}(-\ln y)^{l} d y=\frac{l !}{r^{l+1}}-\frac{l !}{2^{r}} \sum_{p=0}^{l} \frac{\ln ^{p} 2}{r^{l-p+1} p !} .
$$

Полагая $l=m-2$, приходим к утверждению теоремы.

ПримеР 1. Положим $m=2$, что дает

$$
L_{2}=\iint_{W_{2}} \frac{1}{x y}=2 \zeta(2)-\ln ^{2} 2-2 \operatorname{Li}_{2}\left(\frac{1}{2}\right) .
$$

С другой стороны, $L_{2}=I_{0}=\zeta(2)$. Это доказывает формулу Эйлера для дилогарифма в точке $1 / 2$ [1; раздел 1.2], [2; с. 43-45], [16; раздел 1.4]:

$$
\operatorname{Li}_{2}\left(\frac{1}{2}\right)=\sum_{r=1}^{\infty} \frac{1}{r^{2} 2^{r}}=\frac{\zeta(2)}{2}-\frac{\ln ^{2} 2}{2} .
$$


Теперь возьмем $m=3$. Применяя формулу Ландена для трилогарифма в точке $1 / 2$ [16; формула 6.12],

$$
\operatorname{Li}_{3}\left(\frac{1}{2}\right)=\frac{7 \zeta(3)}{8}-\frac{\pi^{2} \ln 2}{12}+\frac{\ln ^{3} 2}{6},
$$

получим

$$
L_{3}=\iiint_{W_{3}} \frac{d x_{1} d x_{2} d x_{3}}{x_{1} x_{2} x_{3}}=6 \zeta(3)-2 \ln ^{3} 2-6 \operatorname{Li}_{3}\left(\frac{1}{2}\right)-6 \ln 2 \operatorname{Li}_{2}\left(\frac{1}{2}\right)=\frac{3}{4} \zeta(3) .
$$

Таким образом, что удивительно, оба числа $L_{3}$ и $I_{1}$ являются рациональными кратными числа $\zeta(3)$.

Наконец, полагая $m=4$ и применяя формулы для $\operatorname{Li}_{2}(1 / 2)$ и $\operatorname{Li}_{3}(1 / 2)$, получим

$$
L_{4}=\frac{4}{15} \pi^{4}-\ln ^{4} 2+\pi^{2} \ln ^{2} 2-21 \zeta(3) \ln 2-24 \operatorname{Li}_{4}\left(\frac{1}{2}\right) .
$$

Теперь обобщим $I_{n}$ на интеграл по многогранник $W_{m}$. Сначала расширим определение полилогарифма $\mathrm{Li}_{s}(z)$, вводя определение кратного полилогарифма

$$
\operatorname{Li}_{s_{1}, \ldots, s_{l}}(z):=\sum_{n_{1}>n_{2}>\cdots>n_{l}>0} \frac{z^{n_{1}}}{n_{1}^{s_{1}} \cdots n_{l}^{s_{l}}} .
$$

Теорема 8. Если $m \geqslant 2$ u $n \geqslant 0$, то интеграл

$$
M_{m, n}:=\int \cdots \int_{W_{m}} \frac{\left(-\ln \left(x_{1} \cdots x_{m}\right)\right)^{n}}{x_{1} \cdots x_{m}} d x_{1} \cdots d x_{m}
$$

равен полиному от многих переменных с рациональными коэффициентами относительно значений $\ln 2, \zeta\left(a,\{1\}_{m+n-a}\right)$ при $m \leqslant a \leqslant m+n u \operatorname{Li}_{b,\{1\}_{c}}(1 / 2)$ nри $b+c \leqslant m+n, b \geqslant 2,0 \leqslant c \leqslant n$.

В явном виде, если $A\left(k_{2}\right):=1 / k_{2}$ ! и если

$$
A\left(k_{2}, \ldots, k_{m}\right):=\frac{1}{k_{2} ! \cdots k_{m} !} \cdot \frac{1}{\left(k_{m}+1\right)\left(k_{m-1}+k_{m}+2\right) \cdots\left(k_{3}+\cdots+k_{m}+m-2\right)}
$$

nрu $m \geqslant 3$, mo

$$
\begin{aligned}
M_{m, n}=m ! n ! & \sum_{\begin{array}{c}
k_{1} \geqslant 0, \ldots, k_{m} \geqslant 0 \\
k_{1}+\cdots+k_{m}=n
\end{array}} A\left(k_{2}, \ldots, k_{m}\right) \\
\times & {\left[\left(k_{2}+\cdots+k_{m}+m-2\right) ! \zeta\left(k_{2}+\cdots+k_{m}+m,\{1\}_{k_{1}}\right)\right.} \\
& \quad-\frac{\ln ^{m+n} 2}{\left(k_{1}+1\right) !(m+n)} \\
& \left.\quad-\left(k_{2}+\cdots+k_{m}+m-2\right) ! \sum_{p=0}^{k_{2}+\cdots+k_{m}+m-2} \frac{\ln ^{p} 2}{p !} \operatorname{Li}_{k_{2}+\cdots+k_{m}+m-p,\{1\}_{k_{1}}}\left(\frac{1}{2}\right)\right] .
\end{aligned}
$$

ДокАЗАТЕЛЬСтво аналогично доказательству теоремы 7 (случай $n=0$ ).

Отметим, что имеет место равенство интегралов $M_{2, n}=I_{n}$. 
В качестве примера применения теоремы 8 получим следующее соотношение между некоторыми кратными значениями полилогарифма и кратными дзета значениями. (Это соотношение также можно вывести из формулы свертки Гёльдера [17; $(7.2)]$.

СлЕДСТВИЕ 7. Если $n \geqslant 0, m o$

$$
\sum_{k=0}^{n} \sum_{p=0}^{n-k} \frac{\ln ^{p} 2}{p !} \operatorname{Li}_{n-k+2-p,\{1\}_{k}}\left(\frac{1}{2}\right)=\frac{1-2^{n+1}}{(n+2) !} \ln ^{n+2} 2+\frac{1}{2} \sum_{k=0}^{n} \zeta\left(n-k+2,\{1\}_{k}\right) .
$$

ДокАЗАТЕЛЬСтво. В теореме 8 возьмем $m=2$ и положим $k_{1}=k$, так что $k_{2}=$ $n-k$. Тогда $A\left(k_{2}\right)=1 /(n-k)$ ! и

$$
\begin{aligned}
M_{2, n}=2 n ! \sum_{k=0}^{n}[\zeta(n & \left.-k+2,\{1\}_{k}\right) \\
& \left.\quad-\frac{\ln ^{n+2} 2}{(k+1) !(n-k) !(n+2)}-\sum_{p=0}^{n-k} \frac{\ln ^{p} 2}{p !} \operatorname{Li}_{n-k+2-p,\{1\}_{k}}\left(\frac{1}{2}\right)\right] .
\end{aligned}
$$

Теперь подставим $M_{2, n}=I_{n}$, и применим теорему 1 , (i) и тождество

$$
\sum_{k=0}^{n} \frac{1}{(k+1) !(n-k) !}=\frac{2^{n+1}-1}{(n+1) !}
$$

ПримеР 2. Случай $n=0$ - это формула Эйлера (22) при $\mathrm{Li}_{2}(1 / 2)$. Полагая $n=1$ и подставляя $\zeta(2,1)=\zeta(3)$, получим соотношение

$$
\mathrm{Li}_{3}\left(\frac{1}{2}\right)+\mathrm{Li}_{2}\left(\frac{1}{2}\right) \ln 2+\mathrm{Li}_{2,1}\left(\frac{1}{2}\right)=-\frac{\ln ^{3} 2}{2}+\zeta(3)
$$

которое является частным случаем уравнения (7.3) в [17]. Подставляя значения $\mathrm{Li}_{2}(1 / 2)$ и $\mathrm{Li}_{3}(1 / 2)$ в $(22)$ и $(23)$, получим формулу

$$
\operatorname{Li}_{2,1}\left(\frac{1}{2}\right)=\sum_{r=2}^{\infty} \frac{1}{r^{2} 2^{r}}\left(1+\frac{1}{2}+\cdots+\frac{1}{r-1}\right)=\frac{\zeta(3)}{8}-\frac{\ln ^{3} 2}{6} .
$$

Наконец, добавляя $\operatorname{Li}_{3}(1 / 2)$, получим суммирование Рамануджана [13; с. 258]

$$
\sum_{r=1}^{\infty} \frac{1}{r^{2} 2^{r}}\left(1+\frac{1}{2}+\cdots+\frac{1}{r}\right)=\zeta(3)-\frac{\pi^{2} \ln 2}{12} .
$$

\section{СПИСОК ЦИТИРОВАННОЙ ЛИТЕРАТУРЫ}

[1] P. Cartier, "Fonctions polylogarithmes, nombres polyzêtas et groupes pro-unipotents", Astérisque, 282, Exp. № 885 (2002), 137-173.

[2] W. Dunham, Euler: The Master of Us All, Dolciani Math. Exp., 22, Mathematical Association of America, Washington, DC, 1999.

[3] S. Zlobin, On a certain integral over a triangle, arXiv: math.NT/0511239. 
[4] F. Beukers, "A note on the irrationality of $\zeta(2)$ and $\zeta(3)$ ", Bull. London Math. Soc., 11:3 (1979), 268-272.

[5] K. S. Kölbig, J. A. Mignaco, E. Remiddi, "On Nielsen's generalized polylogarithms and their numerical calculation", Nordisk Tidskr. Informationsbehandling (BIT), 10:1 (1970), $38-73$.

[6] J. M. Borwein, D. M. Bradley, D. J. Broadhurst, "Evaluations of $k$-fold Euler/Zagier sums: a compendium of results for arbitrary $k$ ", Research paper R5, Electron. J. Combin., 4:2 (1997).

[7] M. Waldschmidt, "Multiple polylogarithms: an introduction", Number Theory and Discrete Mathematics, Proc. Int. Conf. held at Panjab University (Panjab University, Chandigarh, October 2-6, 2000), Trends Math., eds. A. K. Agarwal et al., Birkhäuser Verlag, Basel, 2002, 1-12.

[8] J. Sondow, "Criteria for irrationality of Euler's constant", Proc. Amer. Math. Soc., 131:11 (2003), 3335-3344.

[9] J. Sondow, "Double integrals for Euler's constant and $\ln 4 / \pi$ and an analog of Hadjicostas's formula", Amer. Math. Monthly, 112:1 (2005), 61-65.

[10] J. Ser, "Sur une expression de la fonction $\zeta(s)$ de Riemann", C. R. Acad. Sci. Paris Sér. I. Math., 182 (1926), 1075-1077.

[11] J. Sondow, An infinite product for $e^{\gamma}$ via hypergeometric formulas for Euler's constant, $\gamma$, arXiv: math.CA/0306008, 2003.

[12] J. Sondow, "A faster product for $\pi$ and a new integral for $\ln \pi / 2$ ", Amer. Math. Monthly, 112:8 (2005), 729-734.

[13] B. C. Berndt, Ramanujan's Notebooks, part I, Springer-Verlag, New York, 1985.

[14] N. G. de Bruijn, Asymptotic Methods in Analysis, Dover Publ., New York, 1981.

[15] S. Gradshteyn, I. M. Ryzhik, Table of Integrals, Series, and Products, Academic Press, San Diego, 2000.

[16] L. Lewin, Polylogarithms and Associated Functions, North-Holland Publ., New York, 1981.

[17] J. M. Borwein, D. M. Bradley, D. J. Broadhurst, P. Lisonek, "Special values of multiple polylogarithms", Trans. Amer. Math. Soc., 353:3 (2001), 907-941.

Дж. Сондоу

E-mail: jsondow@alumni.princeton.edu

\section{С. А. Злобин}

Московский государственный университет

им. М. В. Ломоносова

E-mail: sirg_zlobin@mail.ru
Поступило

09.02.2007 